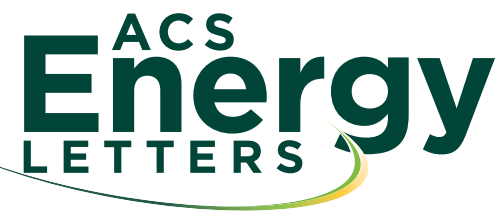

\section{Large-Area Perovskite-Related Copper Halide Film for High-Resolution Flexible X-ray Imaging Scintillation Screens}

\author{
Yang Zhou, ${ }^{\S}$ Xiaojia Wang, ${ }^{\S}$ Tengyue He, ${ }^{\S}$ Haoze Yang, Chen Yang, Bingyao Shao,
} Luis Gutiérrez-Arzaluz, Osman M. Bakr,* Yuhai Zhang,* and Omar F. Mohammed*

Cite This: ACS Energy Lett. 2022, 7, 844-846

Read Online

ABSTRACT: Flexible copper halide films of $400 \mathrm{~cm}^{2}$ area were fabricated with outstanding mechanical stability, excellent film uniformity, nearly $100 \%$ photoluminescence quantum yields, and resistance to water and heat. The re-absorptionfree X-ray imaging scintillators engineered based on these films exhibit superior scintillation performance with a detection limit as low as $48.6 \mathrm{nGy} / \mathrm{s}$ and $17 \mathrm{lp} / \mathrm{mm}$ $\mathrm{X}$-ray imaging resolution, representing the highest imaging resolution for powderbased screens.

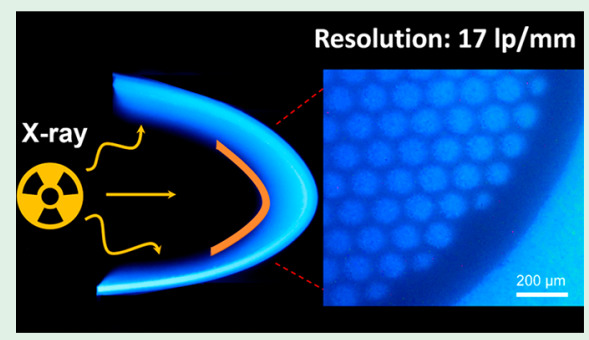

$\mathrm{X}$ -ray imaging scintillation is a critical technology for medical diagnosis as well as industrial and security inspection. ${ }^{1}$ Lead halide perovskite X-ray imaging scintillators have attracted a great deal of attention since the initial report of using all-inorganic $\mathrm{CsPbX}_{3}\left(\mathrm{X}=\mathrm{I}^{-}, \mathrm{Br}^{-}, \mathrm{Cl}^{-}\right.$, or mixed halides) solution-processed nanocrystals as multicolor scintillators. $^{2}$ To guarantee a high light yield, the ideal imaging scintillators should possess a large absorption cross-section for X-ray radiation and simultaneously a large Stokes shift to minimize the re-absorption of the radioluminescence $(\mathrm{RL})$. $^{1,3,4}$ Although the presence of high- $Z$ ions (such as $\mathrm{Cs}, \mathrm{Pb}$, and I) endows perovskites with a considerable X-ray absorption capacity, the significant re-absorption behavior caused by band-edge transitions seriously attenuates the utilizable light yield, leading to a poor scintillation performance. ${ }^{1}$ Moreover, the toxicity of lead and poor air- and light-stability are also serious problems that severely limit their commercialization potential. $^{5,6}$ Therefore, the development of new stable lead-free scintillation materials is urgently needed.

Recently, the lead-free $\mathrm{Cs}_{3} \mathrm{Cu}_{2} \mathrm{I}_{5}$ perovskite-related compound has emerged as a promising candidate for $\mathrm{X}$-ray scintillators because it is eco-friendly and has a high light yield. $^{7-9}$ By virtue of its low-dimensional electronic structure, the $\mathrm{Cs}_{3} \mathrm{Cu}_{2} \mathrm{I}_{5}$ can easily form self-trapped excitons (STEs) due to the lattice's Jahn-Teller distortion. ${ }^{1,10,11}$ The ensuing, large Stokes shift between emission and absorption spectra renders reabsorption-free candidate materials for high-performance X-ray imaging scintillators. Specifically, the $\mathrm{Cs}_{3} \mathrm{Cu}_{2} \mathrm{I}_{5}$ single crystals grown by the Bridgman method showed a high light yield of $\sim 32000$ photons $/ \mathrm{MeV}$, and the $\mathrm{X}$-ray imaging spatial resolution of $6.8 \mathrm{lp} / \mathrm{mm}$ was realized using $\mathrm{Cs}_{3} \mathrm{Cu}_{2} \mathrm{I}_{5}$ film made of ball-milled powder. ${ }^{9,12}$ However, the zero-dimensional (0D) $\mathrm{Cs}_{3} \mathrm{Cu}_{2} \mathrm{I}_{5}$ phase with blue emission is extremely sensitive to moisture, and it readily transforms into $1 \mathrm{D} \mathrm{CsCu}_{2} \mathrm{I}_{3}$ phase with yellow emission under moisture treatment due to the high solubility of CsI in water. ${ }^{13}$ Unfortunately, the light yield of the $\mathrm{CsCu}_{2} \mathrm{I}_{3}$ phase is much lower than that of the $\mathrm{Cs}_{3} \mathrm{Cu}_{2} \mathrm{I}_{5}$ phase, ${ }^{7,14}$ which limits the actual scintillation applications.

Here, the highly stable flexible $\mathrm{X}$-ray scintillation screens with a $400 \mathrm{~cm}^{2}$ area based on the $\mathrm{Cs}_{3} \mathrm{Cu}_{2} \mathrm{I}_{5}$-polydimethylsiloxane (CCI-P) films were realized. Due to their re-absorption-free, high phase purity, and nearly $100 \%$ photoluminescence quantum yields (PLQYs), the films exhibited very promising scintillation properties with a low detection limit of $48.6 \mathrm{nGy} / \mathrm{s}$ and $17 \mathrm{lp} / \mathrm{mm}$ X-ray imaging resolution.

$\mathrm{Cs}_{3} \mathrm{Cu}_{2} \mathrm{I}_{5}$ polycrystalline powders with high phase purity were synthesized by a modified solution process using 2-propanol as the antisolvent (see SI for details, Figures S1-S3). ${ }^{8}$ It is noteworthy that the product yield is more than $85 \%$, which provides a favorable condition for low-cost, large-scale fabrication (Figure S4). The $\mathrm{Cs}_{3} \mathrm{Cu}_{2} \mathrm{I}_{5}$ powders were ground with a mortar and pestle to further refine the particle sizes (Figure S5).

Received: January 11, 2022

Accepted: January 24, 2022 


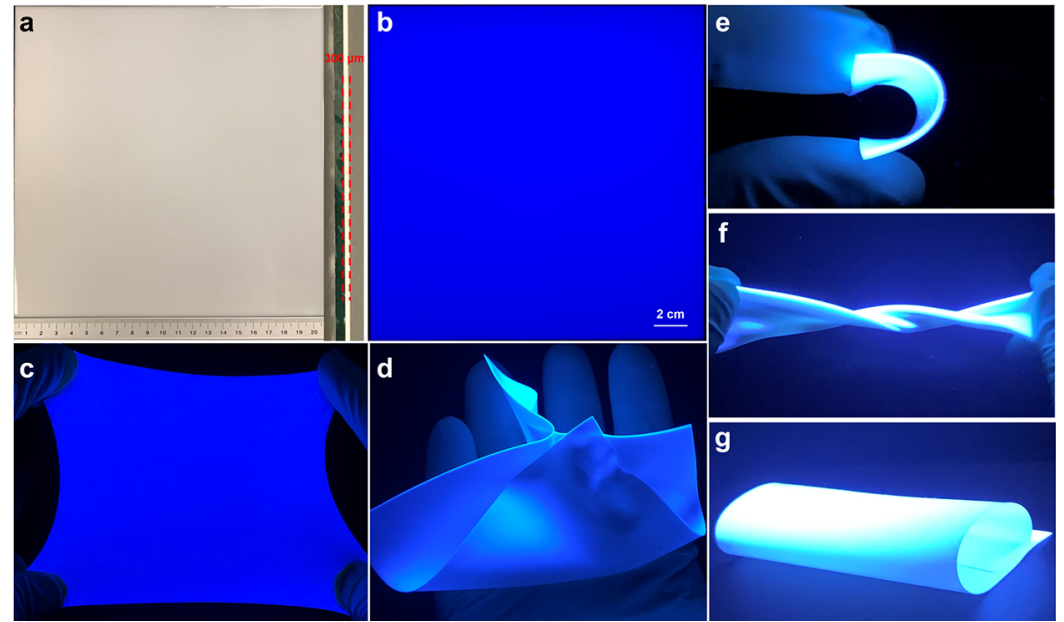

Figure 1. Photographs of the CCI-P-5\% flexible film. The film and its cross section in ambient $(a)$ and under UV excitation (b). (c-g) The film at different mechanical deformations under UV excitation: stretching (c), crumpling (d), bending (e), twisting (f), and folding (g).
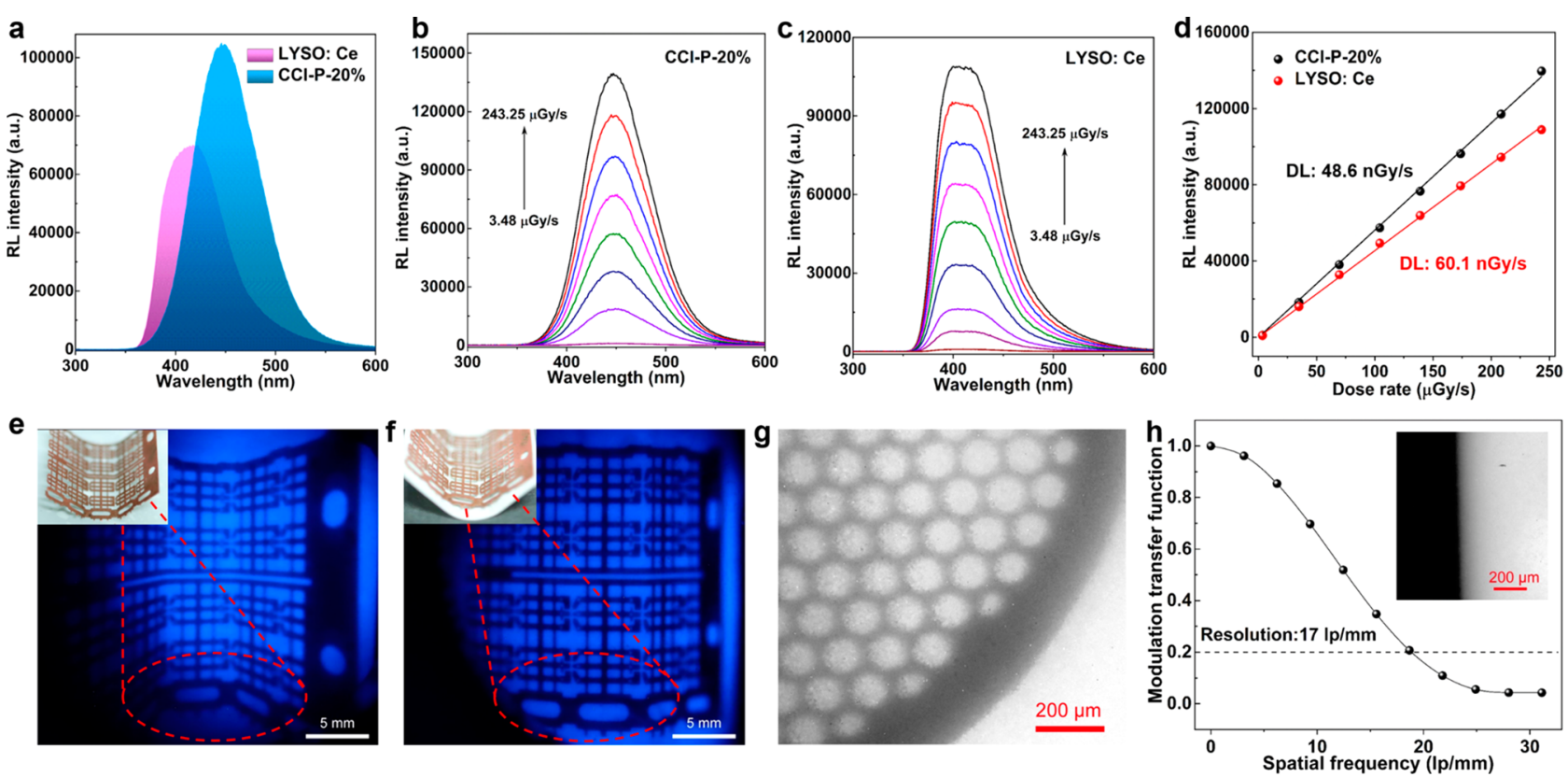

Figure 2. Scintillation properties of the CCI-P- $x \%(x=5,20)$ flexible films. (a) RL spectra of a standard commercial scintillator (LYSO:Ce) wafer and a CCI-P-20\% film. Note that the size and thickness of the wafer and film are the same. Dose rate-dependent RL spectra of the CCI-P20\% film (b) and LYSO: Ce wafer (c). (d) Detection limit (DL) of the CCI-P-20\% film (black line) and LYSO: Ce wafer (red line). X-ray images of flexible copper grid taken by $50 \mu \mathrm{m}$ CCI-P-5\% film under flat (e) and bending (f) states. (g) X-ray image of copper grid with honeycomb structure taken by $50 \mu \mathrm{m}$ CCI-P-5\% film. (h) MTF of the flexible film, measured by the slanted-edge method (inset).

The preparation of CCI-P- $x \%$ (i.e., the mass ratio of $\mathrm{Cs}_{3} \mathrm{Cu}_{2} \mathrm{I}_{5}$ is $\mathrm{x} \%$ ) films is illustrated in Figure S6. Upon addition of ethyl acetate (EA), a very uniform flexible film can be fabricated by the spin-coating or blade-coating method. In contrast, the film without adding EA exhibited poor surface morphology due to the aggregation of $\mathrm{Cs}_{3} \mathrm{Cu}_{2} \mathrm{I}_{5}$ particles (Figure S6). Figure 1a shows the uniform large-area $\left(20 \times 20 \mathrm{~cm}^{2}\right)$ CCI-P-5\% film with a thickness of $300 \mu \mathrm{m}$. Under UV excitation, the film exhibited bright blue emission (Figure $1 \mathrm{~b}$ ). Thanks to the rubber-like nature of polydimethylsiloxane (PDMS), the flexible films have no physical damage in response to mechanical deformations (Figure $1 \mathrm{c}-\mathrm{g}$ ). Furthermore, the XRD pattern of CCI-P-5\% film demonstrated that the $\mathrm{Cs}_{3} \mathrm{Cu}_{2} \mathrm{I}_{5}$ structure remains well after mixing with PDMS (Figure S7). To reveal the photophysical properties of the film, we first investigated the absorption spectra of CCI-P-5\% film, which showed a sharp absorption edge at approximately $325 \mathrm{~nm}$ (Figure S8). Next, we measured the steady-state photoluminescence (PL) spectra. Under UV excitation, the CCI-P-5\% film displayed a broad PL spectrum ranging from 350 to $580 \mathrm{~nm}$, peaking at ca. $445 \mathrm{~nm}$ and with a full width at half-maximum of $81 \mathrm{~nm}$ (Figure S8). The PLQY of CCI-P-5\% film was measured to be about $97.4 \%$. Note that a large Stokes shift of $120 \mathrm{~nm}$ was observed, as shown in Figure S8. To further understand the PL behavior, we measured the PL lifetime for CCI-P-5\% film. As shown in Figure S9, the decay curve of the CCI-P-5\% film can be fitted by a single-exponential function, with a time constant of $969 \pm 8.6 \mathrm{~ns}$. All of the above photophysical characteristics, including long lifetime, large Stokes shift, and broad PL range originate from the STEs. ${ }^{1,11}$ To evaluate the stability of the engineered flexible film for real- 
life applications, we studied its resistivity to water and high temperature. As shown in Figure S10, the blue emission of $\mathrm{Cs}_{3} \mathrm{Cu}_{2} \mathrm{I}_{5}$ powders quickly quenched and evolved to yellow emission under high humidity ( $95 \%)$ treatment, while the CCI-P-5\% film showed outstanding stability and still maintained strong blue emission even when immersed in deionized water. On the other hand, the PL intensity of CCI-P-5\% film remained almost the same under long-time heat treatment (Figure S10).

Both the excellent photophysical properties and high stability of the flexible films manifested a great potential as X-ray imaging scintillators. For instance, the light yield of CCI-P-20\% film was calculated as $\sim 48800$ photons/MeV as compared with the commercial LYSO: Ce scintillator ( 33000 photons/MeV) (Figure 2a). The RL intensity of CCI-P-20\% film was measured under various $\mathrm{X}$-ray doses, showing a well linear correlation with the dose rate of X-rays; and a detection limit of $48.6 \mathrm{nGy} / \mathrm{s}$ was achieved, which was even better than that of commercial LYSO: Ce scintillator $(60.1 \mathrm{nGy} / \mathrm{s})$ (Figure $2 \mathrm{~b}-\mathrm{d}$, Figure S11). We further evaluated the X-ray imaging capability of the flexible films. As shown in Figure 2e,f, the X-ray image of a flexible copper grid recorded by the bending film possesses much better resolution, especially on the edge of copper grid, as compared with that taken by the film under a flat state. Interestingly, the Xray image still maintained high resolution when the film was under stretching state (Figure S12). These confirmed that our flexible films have huge potential for non-flat, flexible target Xray detection. Finally, the excellent resolution ability of the films was demonstrated using a copper grid with honeycomb structure as an imaging object (Figure $2 \mathrm{~g}$ ). ${ }^{15}$ An excellent Xray spatial resolution of $17 \mathrm{lp} / \mathrm{mm}$ was achieved with a modulation transfer function (MTF) value of $20 \%$, representing the highest imaging resolution for powder-based screens (Figure $2 \mathrm{~h}$, Table S1). It is noteworthy that the flexible film based on PDMS not only possesses the homogeneous distribution of $\mathrm{Cs}_{3} \mathrm{Cu}_{2} \mathrm{I}_{5}$ particles that mitigates the scattering of emitted X-ray but also enables the fabrication of a thickness down to $50 \mu \mathrm{m}$, which is critical to achieve high-resolution X-ray imaging.

In summary, we present lead-free large-area flexible $\mathrm{Cs}_{3} \mathrm{Cu}_{2} \mathrm{I}_{5}$ films with high PLQYs, negligible re-absorption, and high air and heat stability. These features make the flexible films excellent $\mathrm{X}$ ray scintillators with a low detection limit of $48.6 \mathrm{nGy} / \mathrm{s}, \sim 113$ times lower than a typical standard dose for X-ray medical imaging. More importantly, the highest X-ray imaging spatial resolution of $17 \mathrm{lp} / \mathrm{mm}$ was achieved, demonstrating the films' promise for medical radiography and security screening applications.

\section{ASSOCIATED CONTENT}

\section{(s) Supporting Information}

The Supporting Information is available free of charge at https://pubs.acs.org/doi/10.1021/acsenergylett.2c00075.

Details of powder preparation, film fabrication, and characterization; Figures S1-S12 and Table S1 (PDF)

\section{AUTHOR INFORMATION}

\section{Corresponding Authors}

Osman M. Bakr - KAUST Catalysis Center, PSE Division, King Abdullah University of Science and Technology, Thuwal 23955-6900, Saudi Arabia; ○ orcid.org/0000-0002-34281002; Email: osman.bakr@kaust.edu.sa

Yuhai Zhang - Institute for Advanced Interdisciplinary Research, University of Jinan, Jinan 250022 Shandong,
China; 이이이.org/0000-0003-0175-0383;

Email: ifc_zhangyh@ujn.edu.cn

Omar F. Mohammed - Advanced Membranes and Porous Materials (AMPM) Center and KAUST Catalysis Center, PSE Division, King Abdullah University of Science and Technology, Thuwal 23955-6900, Saudi Arabia;

(1) orcid.org/0000-0001-8500-1130;

Email: omar.abdelsaboor@kaust.edu.sa

\section{Authors}

Yang Zhou - AMPM and KAUST Catalysis Centers, PSE Division, King Abdullah University of Science and Technology, Thuwal 23955-6900, Saudi Arabia

Xiaojia Wang - Institute for Advanced Interdisciplinary Research, University of Jinan, Jinan 250022 Shandong, China

Tengyue He - AMPM and KAUST Catalysis Centers, PSE Division, King Abdullah University of Science and Technology, Thuwal 23955-6900, Saudi Arabia

Haoze Yang - AMPM and KAUST Catalysis Centers, PSE Division, King Abdullah University of Science and Technology, Thuwal 23955-6900, Saudi Arabia

Chen Yang - AMPM and KAUST Catalysis Centers, PSE Division, King Abdullah University of Science and Technology, Thuwal 23955-6900, Saudi Arabia

Bingyao Shao - KAUST Catalysis Center, PSE Division, King Abdullah University of Science and Technology, Thuwal 23955-6900, Saudi Arabia

Luis Gutiérrez-Arzaluz - AMPM and KAUST Catalysis Centers, PSE Division, King Abdullah University of Science and Technology, Thuwal 23955-6900, Saudi Arabia

Complete contact information is available at:

https://pubs.acs.org/10.1021/acsenergylett.2c00075

\section{Author Contributions}

${ }^{\S}$ Y.Z., X.W., and T.H. contributed equally to this work.

Notes

The authors declare the following competing financial interest(s): O.M.B. is a founder of Quantum Solutions, a nanotechnology company that develops and manufactures quantum dot materials for optoelectronics.

\section{ACKNOWLEDGMENTS}

This work was supported by King Abdullah University of Science and Technology (KAUST).

\section{REFERENCES}

(1) Zhou, Y.; et al. ACS Energy Lett. 2021, 6, 739.

(2) Chen, Q.; et al. Nature 2018, 561, 88.

(3) Wang, J.-X.; et al. ACS Energy Lett. 2022, 7, 10

(4) Shonde, T. B.; et al. ACS Mater. Lett. 2022, 4, 271

(5) Huang, H.; et al. ACS Energy Lett. 2017, 2, 2071.

(6) Chen, B.-A.; et al. ACS Energy Lett. 2017, 2, 342.

(7) Lin, R.; et al. Cell Rep. Phys. Sci. 2021, 2, 100437.

(8) Zhou, J.; et al. Adv. Opt. Mater. 2021, 9, 2002144.

(9) Li, N.; et al. Adv. Opt. Mater. 2022, 2102232.

(10) Luo, J.; et al. Nature 2018, 563, 541.

(11) Jun, T.; et al. Adv. Mater. 2018, 30, 1804547.

(12) Cheng, S.; et al. Phys. Status Solidi RRL 2020, 14, 2000374.

(13) Zhang, F.; et al. Adv. Funct. Mater. 2021, 31, 2105771.

(14) Zhang, M.; et al. Nano Lett. 2021, 21, 1392.

(15) Wang, Z.; et al. Nano Res. 2021, DOI: 10.1007/s12274-0213808-y. 